\title{
Chapter 7 \\ Pulsed Electron-Electron Double Resonance (PELDOR) and Electron Spin Echo Envelope Modulation (ESEEM) spectroscopy in Bioanaly- sis
}

Bode, B. E., and Norman D. G.

\section{Background and history}

Electron Paramagnetic Resonance (EPR) has a long history with the first spectrum being reported in 1945 (Zavoisky 1945) and is adopted widely, in its original continuous wave $(\mathrm{CW})$ form, as a tool for the analysis of both biological systems and materials bearing unpaired electrons. The related technique of "Nuclear Magnetic Resonance" (NMR) developed rapidly thanks to the technological advances that enabled the use of pulsed radiofrequency and Fourier Transformation (FT). In EPR, the challenges posed by needing to generate short and very powerful microwave pulses and the fast relaxation times, of the electron spin, led to a much slower adaptation of pulsed excitation schemes. Nevertheless, early experiments using an NMR spectrometer with a greatly reduced magnetic field had shown the feasibility of pulse EPR (Blume 1958). It was not until the availability of commercial systems in the 1980s that this technique began to be applied more widely outside laboratories focused on the development of instrumentation. Over the last decades the application of pulse EPR has seen a very wide variety of systems studied from biological systems to those of materials science. This development was paralleled by a constant innovation in EPR instrumentation and methodology. Recent success in using arbitrary waveform generators (AWGs) and the commercial implementation and user uptake indicates that EPR is just entering a new era with tremendous opportunities offered by bespoke excitation schemes.

Norman D. G. $(\varangle)$

School of Life Sciences, MSI/WTB Complex, Dow St., Dundee, DD1 5EH, UK.

email: d.g.norman@dundee.ac.uk

Bode, B. E. ( $\square$ )

Biomedical Sciences Research Complex, Centre of Magnetic Resonance and EaStCHEM School of Chemistry, University of St Andrews, St Andrews, KY16 9ST, UK

Email: beb2@st-andrews.ac.uk 


\section{EPR resonance and frequency bands}

The EPR resonance phenomenon relies on the non-degeneracy of the two possible spin states of an unpaired electron in a half-occupied molecular orbital in the presence of a magnetic field. In close analogy to NMR the two energy levels are split by the interaction with the external magnetic field (the Zeeman interaction). The size of the splitting is linearly dependent on the magnetic field strength. Thus, the resonance frequency will be proportional to the magnetic field strength.

Several effects can influence the EPR resonance and result in further features of the signal. Most common are interaction with magnetic nuclei (e.g., ${ }^{14} \mathrm{~N}$ nuclei in common nitroxide spin labels) and anisotropies resulting from different orientations of the molecule having different resonance frequencies. The latter becomes important in powered and frozen solution samples when the orientations do not average quickly as commonly observed in solution (e.g., in high-resolution NMR).

In comparison to high-resolution NMR, where increasing the strength of the magnetic fields used leads to greater sensitivity and chemical shift dispersion, EPR field strengths can have a more complex effect (similar to solid-state NMR). The microwave frequencies corresponding to EPR field strengths used are generally classified into bands (originating from military designation of frequencies with minimal atmospheric absorption when used for radar) and the most noticeable effect of moving to a different band is that the shape of the EPR spectrum can change significantly. A common example is stable nitroxide spin probes: the competing effects of the field-independent coupling to the nitrogen nucleus (hyperfine interaction - in analogy to a J-coupling in NMR the magnitude does not change with the magnetic field) and the field-dependent anisotropic interaction of the electron spin with the magnetic field (Zeeman interaction - in analogy to chemical shifts in NMR the dispersion of signals increases with the magnetic field strength) affects the shape and appearance of the EPR spectrum.

Most advanced EPR applications use immobilised samples (crystals, powders or frozen solutions) which often leads to enhanced relaxation times but also to more complex spectra due to different resonance frequencies and interactions for different anisotropies (Note: The averaging of these anisotropies that has revolutionised solid-state NMR by magic-angle spinning is not practical for EPR as spinning speeds needed would lead to supersonic surface velocities not achievable in these setups.). X-band was the first band to be significantly exploited for pulse EPR ( 9.5 $\mathrm{GHz}$ and $0.3 \mathrm{~T}$ ) (Fig. 7.1A). Generation, manipulation and detection of microwaves was extensively developed for radar applications in World War 2 and components were readily available for this frequency. At this field the spectrum of a nitroxide spin label is dominated by splitting intrinsic to the chemical environment of the unpaired electron (dominated by the nuclear spin of the nitrogen ${ }^{14} \mathrm{~N}, I=1,99.6 \%$ abundance). Although already present at X-band the dispersion due to the different strengths of interaction with the external magnetic field for the different orientations is small. However, as the field is increased this interaction becomes more dominant. 


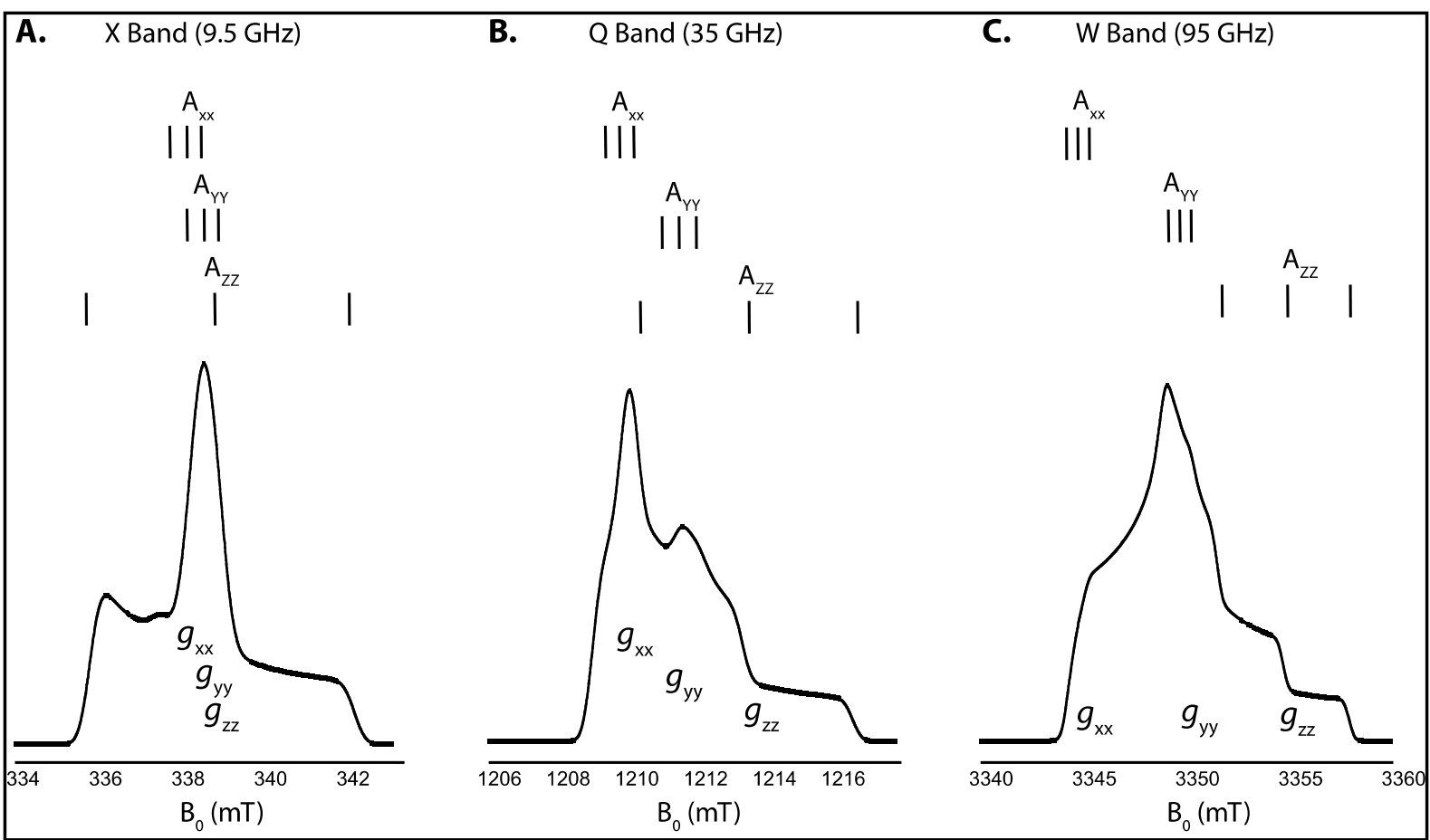

Fig. 7.1 - The absorption spectra of nitroxides at X (A.), Q (B.), and W (C.) band $(9.5,35$ and $95 \mathrm{GHz}$, respectively). Increasing the microwave frequency resolves the $\mathrm{g}$ anisotropy of the sample, broadening the spectra from $\mathrm{X}$ to $\mathrm{W}$ band. The $\mathrm{A}$ and $\mathrm{g}$ anisotropy of a nitroxide are aligned, so as the $\mathrm{g}$ anisotropy of the spectra is resolved, the different $\mathrm{A}$ tensor components occupy different areas of the spectra

Later evolution in microwave technology has allowed for the development and commercial availability of Q (35 GHz), W (95 GHz), and J (260 GHz) band EPR spectrometers, and especially the provision of increased microwave power has greatly enhanced sensitivity. Until recently, commercial EPR spectrometers would only use rectangular pulses, essentially generated by turning the power on and then off. These pulses have the effect of exciting the spectra in a rather complex form giving uneven and not well constrained excitation of the electron spins, described by a sinc function. The incorporation of complex wave forming capability has opened the opportunity for intricate excitation schemes mirroring the successes of NMR in selective excitation and manipulation (Spindler et al. 2013; Doll and Jeschke 2014)

\section{Pulse excitation}

The simplest acquisition scheme in magnetic resonance is detection of a free induction decay (FID). Here, the equilibrium magnetisation of the sample is turned into transverse magnetisation under the influence of a microwave pulse. Monitoring the 
time evolution of this magnetisation, records the FID and its FT gives the spectrum. This is widely applicable in solution NMR. Unfortunately, EPR brings additional challenges such as very broad spectra and fast relaxation times. The FID of a broad spectrum will decay very fast. The use of powerful microwave pulses mandates some dead-time in which the power can dissipate before the weak signal is allowed to reach the sensitive detector (earlier detection would overload and possibly destroy the detector by residual microwave power from the pulse). In combination with the fast decay of the FID, this will often leave very little signal to be detected after the dead-time. To overcome this problem, the signal is recovered by refocusing it as an echo (see section on the spin echo in the following) and the FT of the second half of the echo will give the spectrum. However, this assumes the full spectrum has been excited by the microwave pulse and this is not always the case. If only part of the spectrum is excited the FT will only give the spectrum of the spins excited. The broader the spectrum the shorter and more powerful the excitation needs to be (only very short pulses will excite a broad energy spectrum). In addition, to turn the magnetisation into transverse the overall rotation of the spins must be a quarter circle $\left(90^{\circ}\right.$ or $\left.\pi / 2\right)$ and the rotation angle is given by pulse length multiplied by its magnetic field strength acting on the spins. The magnetic field strength increases with the square root of the microwave power put into the pulse; therefore, a pulse that halves the length (and doubles the width of excitation) needs fourfold higher microwave power. The absolute scaling of the magnetic field strength per microwave wattage depends on the resonator used; for the purpose here, it suffices to say that larger active volumes require more power but allow more sample (and thus more spins) to contribute, boosting sensitivity. Typically pulses in the tens of nanoseconds are used with high power microwave amplification ( $1 \mathrm{~kW}$ at X-band, 150 to $300 \mathrm{~W}$ at Q-band (Polyhach et al. 2012), up to $1 \mathrm{~kW}$ at W-band (Cruickshank et al. 2009)) allowing excitation of about half of the nitroxide spectrum. Usually using shorter and more powerful pulses does increase sensitivity because more spins participate in the experiment and development has led towards the adaptation of ever more powerful spectrometers.

\section{Relaxation}

The relaxation of an excited electron spin is a subject that lies at the heart of EPR spectroscopy with important effects on the EPR spectrum and experiments used to extract information from spin systems. There are essentially two types of relaxation, that are described as $T_{1}$ and $T_{2} . T_{1}$ is also known as spin-lattice relaxation and originates from the interaction of the unpaired electron with surrounding atoms (the lattice). One can imagine $T_{l}$ relaxation as the rate that excited (high energy) spin states relax back to the low energy state. It should be remembered that, under the influence of a magnetic field the population of high and low energy spins are almost equal. The difference in population is described by the Boltzmann equation which is dependent on the energy difference between the two spin states (determined by the magnetic field strength) and the temperature. $T_{2}$ is also called spin-spin or crossrelaxation and is caused by the interaction between the excited electron and other electrons or magnetic nuclei. $T_{2}$ is essentially a dephasing of coherent electron spins 
and does not involve energy dissipation to the lattice. The processes of $T_{l}$ and $T_{2}$ relaxation can be visualised in diagrams such as Fig. 7.2.

One of the consequences of spin relaxation is changes to the spectral linewidth. The linewidth is the inverse of the sum of the relaxation rates. Because relaxation in EPR is very fast compared to NMR, the linewidths of EPR signals are much greater. In solids the linewidths are often dominated by differences in microscopic environment (the inhomogeneous linewidth) and this can also influence the effective $T_{2}$ (in EPR the phase memory time, $T_{m}$, is often given rather than $T_{2}$ to also cover other processes with similar effect but of different nature as $T_{2}$ ). $T_{1}$ is the fundamental restriction on how fast an experiment can be repeated in pulse EPR often making a short time constant beneficial. $T_{2}$ is a fundamental restriction on the persistence of the spin-echo (see section on spin-echo) that restricts the resolution of frequencies and by that also the magnitude of distances that can be measured by pulse EPR often making longer time constants highly beneficial (see section on PELDOR). Manipulation of relaxation using temperature is one of the fundamental techniques used in the RIDME experiment (see section of RIDME).

\section{Relaxation and the spin-echo}

To get around the dead-time problem discussed above, most pulsed EPR experiments make use of the spin echo. A carefully chosen pair of microwave pulses spaced with a timing delay $\tau$ will allow the FID generated by the first pulse but hidden in the deadtime to refocus at delay $\tau$ after the last pulse. If $\tau$ can be chosen long enough the deadtime can be circumvented. This is a technique that has found use in NMR spectroscopy but is almost ubiquitous in pulse EPR. 


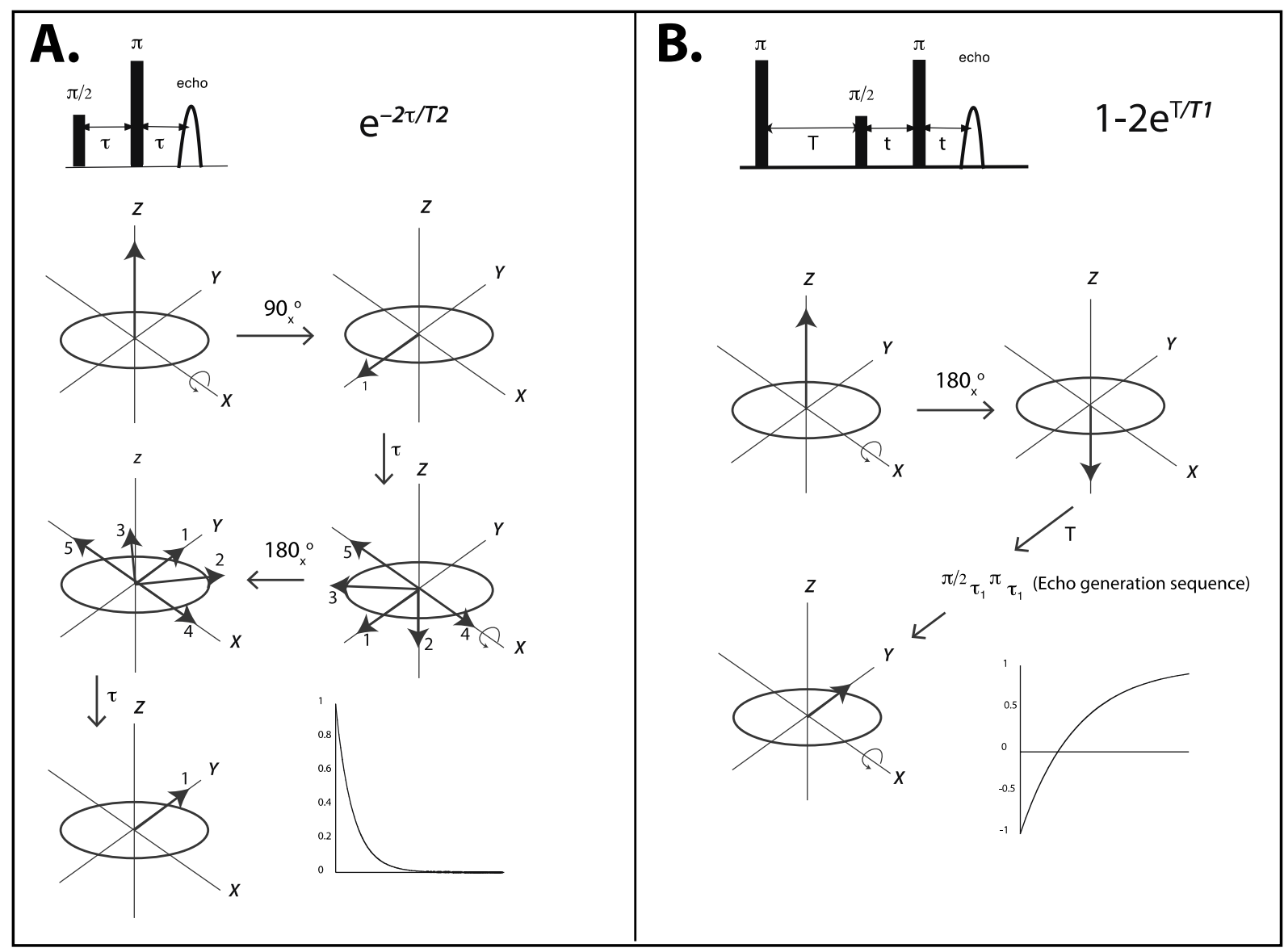

Fig. 7.2 - Diagram illustrating the $T_{2}(\mathrm{~A})$ and $T_{l}(\mathrm{~B})$ modes of relaxation and their measurement. A The pulse sequence shows the first $\pi / 2$ pulse which rotates the electron spin into the transverse orientation in the xy plane. After a time $(\tau)$ the spins have dephased according to their local environment (and corresponding resonance frequency). The application of a $\pi$ pulse then rotates the spin vectors by $180^{\circ}$ around the $\mathrm{x}$-axis. The spins again lie in the xy plane but since the direction and speed of dephasing of individual spins remain the same, after a further delay of time $\tau$, the spins re-phase along the y axis giving rise to an echo. The graph illustrates the decay of phase over time when increasing $\tau$ and the decay is described by the by the exponential factor shown (which includes the time-constant $T_{2}$ ). B: Diagram illustrating the spin inversion and the decay of longitudinal magnetisation. Initially the magnetisation is inverted by a $\pi$ pulse and is now opposite to the thermal equilibrium. Applying the echo sequence leads to an echo pointing into the opposite direction. Incrementing the delay $T$ between the inversion and the echo sequence provides 
increasing time for the spins to return to thermal equilibrium. The exponential recovery of the magnetisation after inversion (called inversion recovery) is recorded as a function of $T$ and shows the characteristic time constant $T_{l}$.

\section{The measurement of dipolar coupling}

One of the most frequently used techniques in EPR, as applied to bioanalysis in recent years, has been the measurement of the dipolar coupling between spin centres and the determination of distances that can be derived from those measurements. Although the pulsed experiment, "pulsed electron-electron double resonance" (PELDOR), also known widely as the "double electron-electron resonance" (DEER) experiment, is the most widespread technique tested and applied, there are other experiments and extensions to PELDOR that are becoming popular. Dipolar coupling between electron spins is a through-space interaction. The frequency of the dipolar coupling is directly related to the distance between the two centres and the frequency is proportional to the inverse of the distance cubed. Alternative approaches have been developed to extract dipolar couplings and to enable distance measurements from systems with two or more unpaired electrons (spins), these include relaxation induced dipolar modulation enhancement (RIDME) (Kulik et al. 2001; Milikisyants et al. 2009), single frequency technique for refocusing (SIFTER) (Jeschke et al. 2000) and double quantum coherence filtered (DQF) (Borbat and Freed 1999) EPR. Each experiment has its place in the toolbox of techniques that can be applied in specific circumstances and are being developed further, especially as hardware capabilities develop.

In a disordered powder sample (this includes frozen solutions) the dipolar coupling between two spins will be determined by the spin-spin distance $r$ and the angle between the distance vector and the external magnetic field $\theta$ (Eq. 7.1):

$\omega_{\mathrm{dd}}=\frac{\mu_{0} \mu_{B}^{2}}{4 \pi \hbar} \frac{g_{\mathrm{A}} g_{\mathrm{B}}}{r^{3}}\left(1-3 \cos ^{2} \theta\right)$

where $g_{\mathrm{A}}$ and $g_{\mathrm{B}}$ are the $g$-values of the two coupled spins and assumed to be isotropic, $\mu_{0}$ is the vacuum permeability, $\mu_{\mathbf{B}}$ is the Bohr magneton, $\hbar$ is the reduced Planck constant.

\section{Pulsed Electron-Electron Double Resonance (PELDOR) experiment}

In its basic form the PELDOR experiment is a two-frequency experiment that takes advantage of the broad EPR spectra to excite separate populations of spins. Fig. 7.3 shows the sequence of microwave pulses used in 4-pulse PELDOR. 


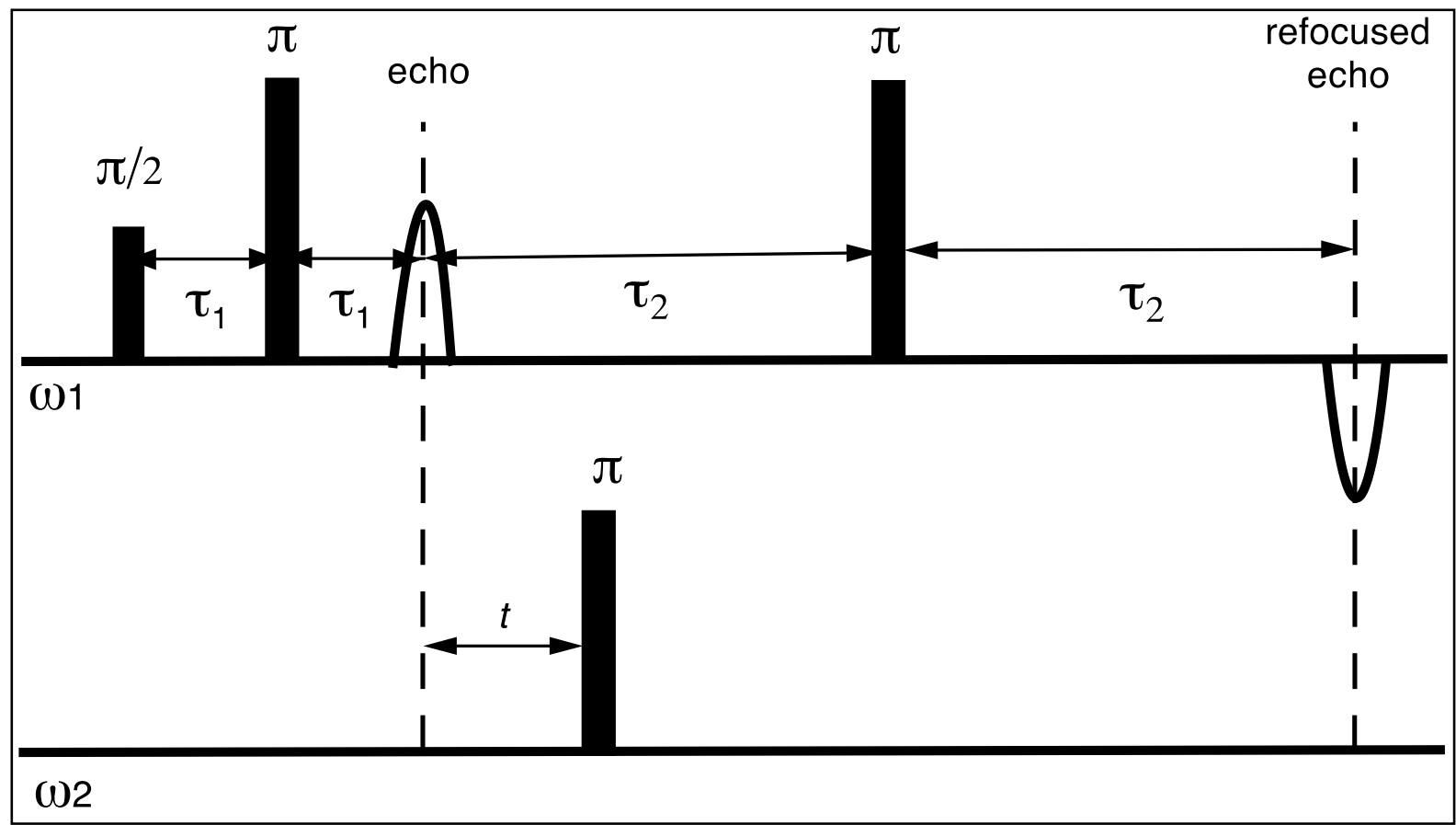

Fig. 7.3 - The pulse sequence for the 4-pulse PELDOR experiment. The microwave pulses are represented by solid rectangles on the two, time axes representing the two frequencies $\omega 1$ and $\omega 2$. Frequency $\omega 1$ is showing the echo-forming pulse sequence and subsequent refocusing by a further pulse. The pulse with frequency $\omega 2$ is inverting the second set of spins at a defined delay with respect to the primary echo. This delay is incremented and the intensity of the refocused echo with respect to this delay is recorded.

The top line of Fig. 7.3 shows the pulses, at what is called the observer or detection frequency $(\omega 1)$, and first develops a spin echo using the 2-pulse sequence that was previously described. Shown on the lower line is the second frequency $(\omega 2)$ pulse, known as the pump pulse, which is set to excite a separate population of spins at a variable time $t$ after the echo from the top line forms. The pump pulse is moved along by small time increments as the experiment develops. This pulse switches the orientation of the second set of spins and sets the spin-spin interaction, via dipolar coupling. The interaction between the spins changes the resonance frequency of the first set of spins according to the interaction frequency. The time $t$ of the pump pulse determines the net time the coupling is switched. A further pulse is used at the observer frequency to again refocus the signal and so develop a spin echo (called refocused echo) shown at the end of the diagram. The final echo is detected and its intensity is recorded for each increment of the pump pulse. The signal recorded from this experiment, is echo intensity against time and if pairs of spins are situated at a 
fixed distance from each other, in the molecule under study, then a damped oscillation will be observed (Fig. 7.4 A). The background signal derived from the interaction between spins on different molecules can be fitted to a simple model based on a random (homogeneous) 3D distribution of molecules in the sample, and that contribution removed (Fig. 7.4 B). As sketched above, the frequency of this echo oscillation reports on the dipolar coupling and consequently the distance (eq. 1). A short distance would give high frequency, short period, amplitude modulations. If the distance between the spins was longer, then the frequency of the amplitude modulation would be smaller and the wavelength longer. The more similar two distances are, the more similar their frequencies become. This means to recover long distances or small differences, then long times of $t$ must be recorded (Ackermann and Bode 2018).

The recorded echo amplitude modulation can be read off directly and converted into a distance (this can be done from the FT of the oscillation, see Fig. 7.4 C), although more powerful techniques use mathematical fitting algorithms to extract not only a distance measurement but also a measure of the distance distribution (Jeschke et al. 2006) that exists between the two spins (Fig. 7.4 D). It should be noted that the depth of the oscillation ( $\Delta$ in Fig. 7.4 B) depends on the average excitation of partner spins. This in turn depends on the probability of exciting a spin under the second frequency pulse and the number of partner spins in the molecule or complex. Carefully performed these experiments can be used to count the number of interacting spins and determine oligomerisation equilibria (Bode et al. 2007) (Ackermann et al. 2015).

The typical PELDOR experiment, using $100 \mu \mathrm{M}$ spin concentration and $100 \mu \mathrm{L}$ sample, for not too challenging distances (up to $60 \AA$ ), will take several hours at Xband and mere minutes at $\mathrm{Q}$ and $\mathrm{W}$-band (this assumes high power setups). The range of distances between spins, that can be measured is from around $15 \AA$ up to about $160 \AA$ depending on the relaxation rate $\left(\mathrm{T}_{2}\right)$ of the spin echo which is a consequence of the chemical environment present in both the molecule under study and the solvent used in the sample. The factor dictating how far a measurement can go is largely the persistence of the refocussed echo. The PELDOR experiment, in its normally used form, is a constant time experiment and the time axis, on which the decaying signal is gathered, is set by the delays ( $\tau_{2}$ on Fig. 7.3). As one increases the length of the delay $\tau_{2}$, then one pushes the refocussed echo farther out and allows one to gather data over a longer period of time. The echo decreases as the time period $\tau_{2}$ is increased due to relaxation that cannot be refocussed and so there is a limit to the delay $\tau_{2}$ and the time axis of the recorded data. To measure a frequency or a distance from the decaying signal one must record enough data to capture sufficient oscillations in the signal. As the distance between the spins increases so the wavelength of the echo oscillation increases (eq. 1) and one needs to record more time. At some point the echo becomes too small to be recorded and this fixes the limitation to the measurable distance. 


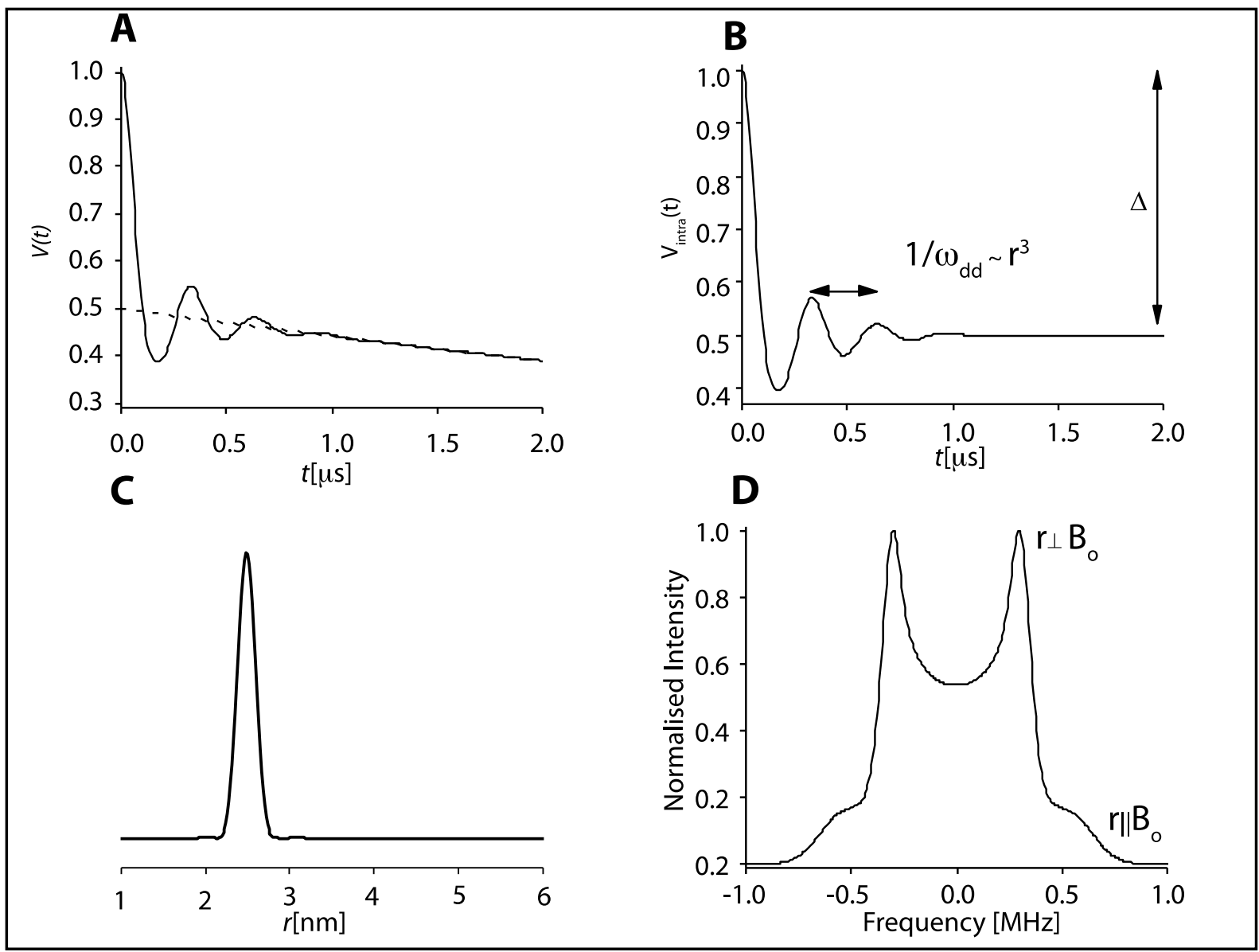

Fig. 7.4 - Data derived from the PELDOR experiment and its treatment to extract distance data. A. Raw data from measurement of the echo amplitude modulation, the dashed line indicates a fitted background signal. B. The background-corrected data from the PELDOR experiment with indication of a single oscillation whose frequency is proportional to the cube of the distance. C. The background corrected PELDOR data can be transformed to reveal the distance and the distance distribution between spins. D. The Fourier transform of the background-corrected PELDOR data (a so-called Pake pattern) reveals the frequency distribution of the dipolar spinspin interaction.

\section{Single frequency experiments}


The double frequency experiments such as the PELDOR experiment have some limitations that arise from the limited spectral width that is accessible on most present-day spectrometers. Because the two frequencies must both lie within the resonant cavity, one is generally forced into a compromised sensitivity. If one can use a single frequency then it can be placed at the most resonant position within the cavity (with a high-quality factor), maximising sensitivity. The width of EPR spectra often makes these experiments practically inferior to PELDOR (judging from uptake by EPR practitioners). The limited excitation bandwidth of the pulses leads to incomplete refocusing and thus, all intricate multi-pulse schemes will suffer from a mixture of signals arising from different coherence transfer pathways where one or more pulses have not been equally effective. Nevertheless, for narrow EPR spectra as e.g. triarylmethyl (TAM) radicals, SIFTER has been successfully applied (Meyer et al. 2018).

In an early adaptation of NMR methodology Freed and co-workers have shown that DQF methods allow extraction of dipolar interactions at superior sensitivity (Yang et al. 2012). However, the methodology is instrumentally demanding (requiring very short microwave pulses and accurate phase cycles) and the robustness of PELDOR methods has led to most applications relying on the double frequency method.

\section{Relaxation Induced Dipolar Modulation Enhancement (RIDME)}

In PELDOR we have seen that two frequencies are used to excite two separate groups of spins that then interact to give an oscillating intensity to the spin echo. RIDME is an experiment that, rather than using a second frequency, uses the naturally fast relaxation, of at least one component (spin) to set the coupling interaction. In RIDME the second frequency of PELDOR is essentially substituted by a time delay in which the second spin is allowed to flip by spontaneous relaxation and substitutes for the active spin flip caused by the pump pulse on the second frequency. This relaxation delay in RIDME is moved across the first echo and then the echo oscillation is either detected or, in a more advanced form, the echo is reformed, removing it from the dead-time (Fig. 7.5). Setting up a RIDME experiment requires knowledge of the $T_{l}$ relaxation rate and the tailoring of that rate by suitable choice of temperature ( $T_{l}$ is generally strongly affected by temperature). RIDME data can be analysed very similarly to PELDOR to extract distance distributions (Abdullin et al. 2015) and numbers of interacting spins (Giannoulis et al. 2017). The RIDME experiment has been shown to have great utility and potential for measurements involving at least one suitable paramagnetic metal ion and another spin. In particular, RIDME has been used successfully in systems containing $\mathrm{Cu}^{2+}$ and $\mathrm{Gd}^{3+}$ ions as well as $\mathrm{Mn}^{2+}$ and $\mathrm{Fe}^{3+}$.(Meyer and Schiemann 2016; Meyer et al. 2016; Abdullin et al. 2015; Akhmetzyanov et al. 2016; Kaur et al. 2018) 


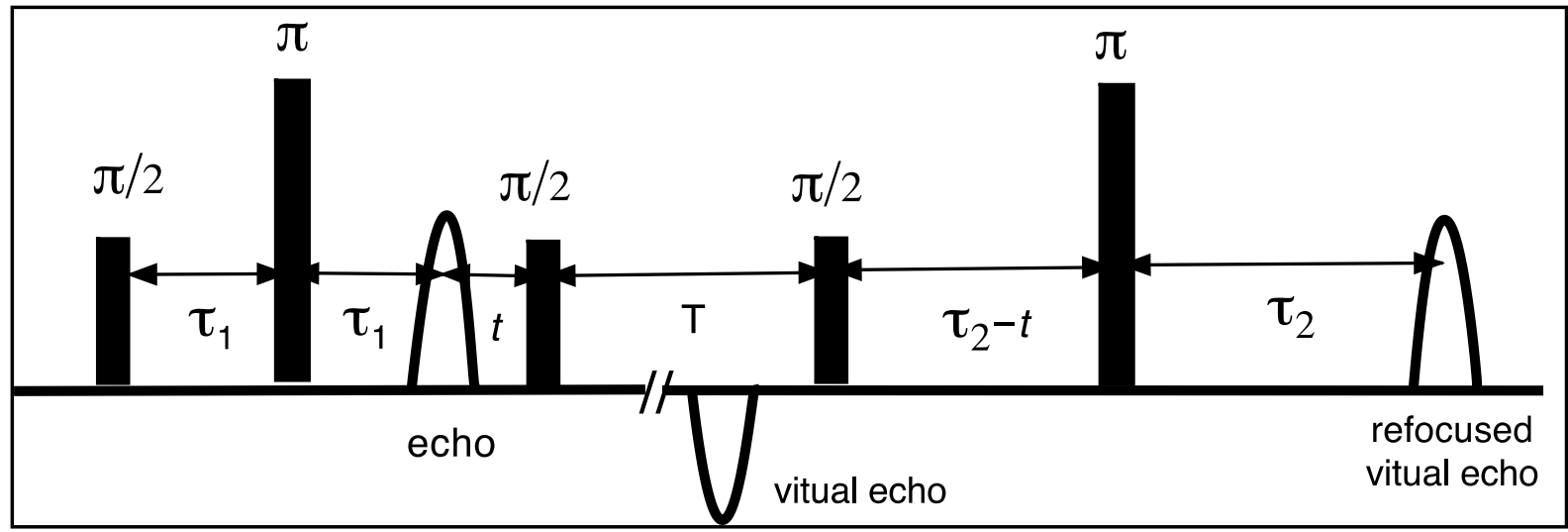

Fig. 7.5 - The pulse sequence for the 5-pulse RIDME experiment. The microwave pulses are represented by solid rectangles on the time axes. The time delay $(t)$ is incremented and the intensity of the refocused echo with respect to this delay is recorded. This sequence is easiest rationalised by replacing the second frequency $\pi$ pulse of the PELDOR sequence with a $\pi / 2-\mathrm{T}-\pi / 2$ sequence that is called "mixing block". During this time the dipolar coupling is set by the B spins flipping an odd number of times (for an even number of flips there is no net effect).

As well as offering an alternative to PELDOR, at some frequencies RIDME can be used for combinations of spins that, with extremely wide spectral widths and separations, would not be suitable for PELDOR due to the spectra being so far separated that they cannot simultaneously be excited by the pulses. In fact, RIDME can use a deliberately reduced resonator bandwidth thereby boosting sensitivity even further. One of the severe problems to overcome in RIDME has been the production of echo oscillations containing ESEEM derived signal (see ESEEM section), from either proton or deuterons in the vicinity. These can currently be addressed by either averaging different pulse delay timings (Keller et al. 2016) (effectively averaging the oscillations) or deconvoluting them from experiments that only show unwanted ESEEM and those showing both ESEEM and RIDME effects. (Abdullin et al. 2015)

\section{The use of shaped pulses}

The advent of arbitrary waveform generators (AWG) that allow generation of arbitrary amplitude, phase and frequency of microwaves on sub-nanosecond timescales has brought many concepts from NMR into reach. While the limited excitation bandwidth and excitation sidebands of rectangular microwave pulses have been severely limiting in the use of multi-pulse excitation, the use of shaped and chirped (frequency swept) pulses allows one to boost sensitivity and execute bespoke control of spin population excitation. Quite excitingly it might bring the NMR paradigm to EPR. As explained when introducing pulse excitation, the EPR echo is the refocused FID of the part of the EPR spectrum that has been excited. Thus, the echo is mostly recorded as a function of magnetic field to obtain a spectrum despite the 
limited excitation. In practice, that means that using FID detection (in NMR) or echoes that represent the excitation of the full spectrum (in solid-state NMR) the spectrum dimension can be obtained from a single experiment and subsequent FT. In common echo detected EPR each point on the axis of the spectrum is a separate experiment. With AWGs now allowing the excitation of the full spectrum, the latter can be retrieved from either the FID (dead-time permitting) or the second half of the echo (i.e., refocused FID).

This gives the EPR spectrum from FT (similarly to the direct dimension in NMR) and allows, for instance, measuring relaxation times by only sampling the relaxation delays and still obtaining information for all parts of the spectrum. Currently, if one wants to know relaxation times as a function of the spectral position in EPR, both the field and the relaxation delays must be sampled individually in two-dimensional experiment which can easily be prohibitively expensive in terms of measurement time and cryogen consumption. One striking example how AWGs enable this concept is the 2D SIFTER experiment (Doll and Jeschke 2016) (Bowen et al. 2018).

\section{Experimental conditions (sample size, concentration, cryoprotectant and sol- vent deuteration)}

The small energies involved in the spin-transitions underpinning magnetic resonance experiments make the method non-destructive and highly reproducible but also less sensitive than, for example optical spectroscopy. Magnetic resonance often compensates by using repeated signal acquisition and averaging. The signal will increase linearly with increasing repetition but unfortunately the measured noise also increases, with the square root of the number of experiments. In consequence, the signal-to-noise ratio (SNR) that determines what can be resolved from the data will improve only with the square root of the number of experiments. This means that one has to increase the acquisition time by a factor of four in order to merely double the signal quality. This shows why the optimisation of the signal is paramount as compensation of a poor signal by longer measurement is severely limited. Sample sizes are normally chosen to fill the active volume of the probe head used and larger volume samples ( $\sim 3 \mathrm{~mm}$ tubes) have brought significant advances at Qand W-band (Polyhach et al. 2012; Cruickshank et al. 2009). However, probe heads with smaller active volumes generally provide better SNR per volume and so it is usually best to take the smallest active volume probe head that will still accommodate the sample available. If the sample amount is limiting a probe head with a small active volume will have higher SNR than a probe head with larger active volume used with the same sample (better absolute sensitivity). On the other hand, if sample concentration is limiting, it is advisable to fill a large volume probe rather than a small volume probe head as more spins will be contributing to the experiment (better concentration sensitivity). Distance measurements by EPR are performed in immobilised samples for a number of reasons, one being that rapid tumbling in solution would average the dipolar interaction to zero. Frozen aqueous buffers tend to crystallise and this causes high local concentration or aggregation of the solute biomolecules. Extreme local concentration leads to a drastic shortening of the echo decay (or relaxation) times and considerably limits the measurable distances. It is 
standard practice to use cryoprotectants such as ethylene glycol or glycerol to inhibit ice crystal formation and allow the formation of a frozen glassy solution. The effect on biological function and biomolecular stability has to be carefully considered and concentrations between $20 \%$ and $60 \%$ have been found to be useful without impairing function in most cases. Even if aggregation can be avoided, the length of traces that can be recorded will be limited by the echo lifetime and this will be limited by the sample concentration and by the type of magnetic nuclei surrounding the electron spins. The relaxing effects of nearby protons can be decreased by deuteration. Replacing the protons of the buffer and cryoprotectant by deuterons is done routinely and can significantly increase the distances measurable. The longest distances measured have been achieved in systems where all the protons in the protein are replaced by deuterons as well, although this requires special procedures for the production of the samples. The sample concentration increases relaxation and the PELDOR background decay (Fig. 7.4 part A) (Jeschke and Polyhach 2007). This limits the measurable distances and leads to lower concentrations required when long distances are to be measured.

\section{Some examples of PELDOR studies}

One striking example of PELDOR application is the measurement of distances up to $16 \mathrm{~nm}$ in the tetradecameric membrane protein GroEL (Schmidt et al. 2016b). This experiment requires to minimise dephasing due to $T_{2}$ and avoidance interference effects by multiple spin labels (Giannoulis et al. 2013; von Hagens et al. 2013) (in a homo-tetradecamer all 14 subunits are identical and the complex yields 14 labels when each monomer is labelled). This achievement was a combination of protein deuteration (Bowman et al. 2014) (Ward et al. 2010) (El Mkami and Norman 2015), reducing the excitation strength of the pump pulse and reducing spin labelling. The effects of the latter two have been shown systematically on the homoheptameric mechanosensitive channel of small conductance (MscS). Biological mechanosensing is a very active field of research with implications for health and disease. In the search for a structural basis of the gating of the channel PELDOR was employed to unravel the MscS gating state in frozen solution (Pliotas et al. 2012) (Ward et al. 2014). During these investigations, high quality-distance distributions could be obtained when the simultaneous pumping of multiple spins was deliberately suppressed by weakening the second frequency pulse in PELDOR (Valera et al. 2016). When reducing the number of spins per protein monomer by a procedure blocking some cysteine residues with a non-magnetic analogue, the data quality was improved even further (Ackermann et al. 2017). Ultimately PELDOR has informed structural biology about the solution state of MscS and contributed to the overall understanding of the role of lipids in mechanosensation (Pliotas et al. 2015).

While PELDOR and other dipolar techniques are widely explored for the measurement of distances opportunities for scrutinising multimerisation equilibria are emerging. Recent examples include concentration dependent dimerisation of influenza proteins (Georgieva et al. 2015) (Kerry et al. 2014) as well as dimerisation of 
fibril modelling peptides (Kotler et al. 2019) that can be rationalised by multimerisation studies (Schmidt et al. 2016a).

\section{ESEEM}

While EPR spectroscopy is based on observing changing populations of energy levels of the electron spin, there are also transitions of nuclear spins. At low magnetic field, where the anisotropy of the electron nuclear (hyperfine) interaction is not small compared to the Zeeman interaction, electron and nuclear spins can both flip simultaneously under a microwave pulse. The probability of this depends on pulse timings and interaction strengths and as a consequence, when the delay times between pulses are changed, an oscillatory signal occurs. In the simplest case of changing $\tau$ in a Hahn echo sequence one can observe the echo decay (caused by $T_{2}$ relaxation) being superimposed with a modulation with nuclear Larmor frequencies. This Electron Spin Echo Envelope Modulation (ESEEM) (Van Doorslaer 2017) is a powerful technique that has been shown to provide a wealth of information on the interaction of the electron spin with nuclei which are in close vicinity. The fact that we can detect ESEEM type interactions means we can interrogate the chemical nature of the material that surrounds an unpaired electron. Conversely, as ESEEM originates from changing pulse delays leading to oscillatory signals, it becomes likely to occur as a side product of other pulse experiments.

There are a large number of experimental variants used to study the ESEEM effect, also extending to multiple dimensions, and tailored to specific situations allowing the study of paramagnetic centres including metals and stable radicals. These are covered in several excellent reviews (Van Doorslaer 2017; McCracken 2011) (Deligiannakis et al. 2000) (Thomas Prisner 2001). In the context of long-range structural constraints there are two important aspects. Firstly, ESEEM can be used to study interactions with solvent nuclei. This is exploited to determine topologies upon deuterium exchange of buffers and/or membranes or even protein deuteration. Comparing deuterium ESEEM modulation depths at different levels of deuteration of the sample (protein or buffer/detergent/membrane) allows a semiquantitative assessment of solvent accessibility or membrane exposure. Secondly, unwanted ESEEM modulations can appear in any pulse EPR experiment where electron spins experience pulses with incremented delays. In PELDOR this is circumvented by diminishing the spectral overlap between the two frequencies used in the experiment, albeit the excitation sidebands can overlap causing some residual ESEEM. As moving frequencies further apart is not always feasible, schemes averaging the ESEEM modulation are often used. This is possible as the ESEEM artefacts are often caused by solvent nuclei and couplings are commonly small leading to modulation with nuclear Larmor frequency. Setting pulse delays to a multiple of the inverse Larmor frequency and averaging them over one modulation period has largely abolished this problem for PELDOR. In RIDME all pulses are at single frequency making ESEEM often much more severe. There are different approaches to tackle this problem, including averaging, (Keller et al. 2016) deconvolution of reference experiments (Abdullin et al. 2015) and reducing excitation strengths 
(Giannoulis et al. 2018). This remains a very active field of research. However, the use of high fields will be particularly beneficial (Kaur et al. 2018).

One landmark study exploiting ESEEM to infer changing solvent accessibility during protein folding was performed on plant light harvesting complexes and allowed understanding water accessibility (Volkov et al. 2009)as well as monitoring refolding of the integral membrane (Dockter et al. 2009) protein. Similarly, water accessibility and interactions with phospholipids could be traced by ESEEM on spinlabelled potassium channel KcsA (Cieslak et al. 2010). This information can be used to model the phosphate interaction surface of the integral membrane protein on a crystallographic structure. Both studies demonstrate the value of ESEEM for spin-labelled proteins and add to the value hyperfine methods are known to have for intrinsic paramagnetic centres.

\section{References}

Abdullin D, Duthie F, Meyer A, Muller ES, Hagelueken G, Schiemann O (2015) Comparison of PELDOR and RIDME for Distance Measurements between Nitroxides and Low-Spin Fe(III) Ions. J Phys Chem B 119 (43):13534-13542. doi:10.1021/acs.jpcb.5b02118

Ackermann K, Bode BE (2018) Pulse EPR distance measurements to study multimers and multimerisation. Mol Phys 116 (12):1513-1521. doi:10.1080/00268976.2017.1421324

Ackermann K, Giannoulis A, Cordes DB, Slawin AMZ, Bode BE (2015) Assessing dimerisation degree and cooperativity in a biomimetic small-molecule model by pulsed EPR (vol 51, pg 5257, 2015). Chem Commun 51 (84):15472-15472. doi:10.1039/c5cc90439k

Ackermann K, Pliotas C, Valera S, Naismith JH, Bode BE (2017) Sparse Labeling PELDOR Spectroscopy on Multimeric Mechanosensitive Membrane Channels. Biophys J 113 (9):1968-1978. doi:10.1016/j.bpj.2017.09.005

Akhmetzyanov D, Ching HYV, Denysenkov V, Demay-Drouhard P, Bertrand HC, Tabares LC, Policar C, Prisner TF, Un S (2016) RIDME spectroscopy on high-spin Mn2+ centers. Phys Chem Chem Phys 18 (44):30857-30866. doi:10.1039/c6cp05239h

Blume RJ (1958) Electron Spin Relaxation Times in Sodium-Ammonia Solutions. Phys Rev 109 (6):1867-1873. doi:DOI 10.1103/PhysRev.109.1867

Bode BE, Margraf D, Plackmeyer J, Durner G, Prisner TF, Schiemann O (2007) Counting the monomers in nanometer-sized oligomers by 
pulsed electron - Electron double resonance. J Am Chem Soc 129 (21):6736-6745. doi:10.1021/ja065787t

Borbat PP, Freed JH (1999) Multiple-quantum ESR and distance measurements. Chem Phys Lett 313 (1-2):145-154. doi:Doi 10.1016/S0009-2614(99)00972-0

Bowen AM, Erlenbach N, van Os P, Stelzl LS, Sigurdsson ST, Prisner TF (2018) Orientation Selective 2D-SIFTER Experiments at X-Band Frequencies. Appl Magn Reson 49 (12):1355-1368. doi:10.1007/s00723-018-1057-3

Bowman A, Hammond CM, Stirling A, Ward R, Shang WF, El-Mkami H, Robinson DA, Svergun DI, Norman DG, Owen-Hughes T (2014) The histone chaperones Vps75 and Nap1 form ring-like, tetrameric structures in solution. Nucleic Acids Res 42 (9):6038-6051. doi:10.1093/nar/gku232

Cieslak JA, Focia PJ, Gross A (2010) Electron Spin-Echo Envelope Modulation (ESEEM) Reveals Water and Phosphate Interactions with the KcsA Potassium Channel. Biochemistry-Us 49 (7):14861494. doi:10.1021/bi9016523

Cruickshank PAS, Bolton DR, Robertson DA, Hunter RI, Wylde RJ, Smith GM (2009) A kilowatt pulsed 94 GHz electron paramagnetic resonance spectrometer with high concentration sensitivity, high instantaneous bandwidth, and low dead time. Rev Sci Instrum 80 (10). doi:Artn 103102

$10.1063 / 1.3239402$

Deligiannakis Y, Louloudi M, Hadjiliadis N (2000) Electron spin echo envelope modulation (ESEEM) spectroscopy as a tool to investigate the coordination environment of metal centers. Coordin Chem Rev 204:1-112. doi:Doi 10.1016/S0010-8545(99)00218-0

Dockter C, Volkov A, Bauer C, Polyhach Y, Joly-Lopez Z, Jeschke G, Paulsen H (2009) Refolding of the integral membrane protein light-harvesting complex II monitored by pulse EPR. P Natl Acad Sci USA 106 (44):18485-18490. doi:10.1073/pnas.0906462106

Doll A, Jeschke G (2014) Fourier-transform electron spin resonance with bandwidth-compensated chirp pulses. J Magn Reson 246:18-26. doi:10.1016/j.jmr.2014.06.016

Doll A, Jeschke G (2016) EPR-correlated dipolar spectroscopy by Q-band chirp SIFTER. Phys Chem Chem Phys 18 (33):23111-23120. doi:10.1039/c6cp03067j

El Mkami H, Norman DG (2015) EPR Distance Measurements in Deuterated Proteins. Method Enzymol 564:125-152. doi:10.1016/bs.mie.2015.05.027 
Georgieva ER, Borbat PP, Norman HD, Freed JH (2015) Mechanism of influenza A M2 transmembrane domain assembly in lipid membranes. Sci Rep-Uk 5. doi:ARTN 11757

10.1038/srep11757

Giannoulis A, Motion CL, Oranges M, Buhl M, Smith GM, Bode BE (2018) Orientation selection in high-field RIDME and PELDOR experiments involving low-spin Co-II ions. Phys Chem Chem Phys 20 (4):2151-2154. doi:10.1039/c7cp07248a

Giannoulis A, Oranges M, Bode BE (2017) Monitoring Complex Formation by Relaxation-Induced Pulse Electron Paramagnetic Resonance Distance Measurements. Chemphyschem 18 (17):2318-2321. doi:10.1002/cphc.201700666

Giannoulis A, Ward R, Branigan E, Naismith JH, Bode BE (2013) PELDOR in rotationally symmetric homo-oligomers. Mol Phys 111 (18-19):2845-2854. doi:10.1080/00268976.2013.798697

Jeschke G, Chechik V, Ionita P, Godt A, Zimmermann H, Banham J, Timmel CR, Hilger D, Jung H (2006) DeerAnalysis2006 - a comprehensive software package for analyzing pulsed ELDOR data. Appl Magn Reson 30 (3-4):473-498. doi:10.1007/Bf03166213

Jeschke G, Pannier M, Godt A, Spiess HW (2000) Dipolar spectroscopy and spin alignment in electron paramagnetic resonance. Chem Phys Lett 331 (2-4):243-252. doi:Doi 10.1016/S00092614(00)01171-4

Jeschke G, Polyhach Y (2007) Distance measurements on spin-labelled biomacromolecules by pulsed electron paramagnetic resonance. Phys Chem Chem Phys 9 (16):1895-1910. doi:10.1039/b614920k

Kaur H, Abreu B, Akhmetzyanov D, Lakatos-Karoly A, Soares CM, Prisner T, Glaubitz C (2018) Unexplored Nucleotide Binding Modes for the ABC Exporter MsbA. J Am Chem Soc 140 (43):14112-14125. doi:10.1021/jacs.8b06739

Keller K, Doll A, Qi MA, Godt A, Jeschke G, Yulikov M (2016) Averaging of nuclear modulation artefacts in RIDME experiments. J Magn Reson 272:108-113. doi:10.1016/j.jmr.2016.09.016

Kerry PS, Turkington HL, Ackermann K, Jameison SA, Bode BE (2014) Analysis of Influenza A Virus NS1 Dimer Interfaces in Solution by Pulse EPR Distance Measurements. J Phys Chem B 118 (37):10882-10888. doi:10.1021/jp508386r

Kotler SA, Tugarinov V, Schmidt T, Ceccon A, Libich DS, Ghirlando R, Schwieters CD, Clore GM (2019) Probing initial transient oligomerization events facilitating Huntingtin fibril nucleation at 
atomic resolution by relaxation-based NMR. P Natl Acad Sci USA 116 (9):3562-3571. doi:10.1073/pnas.1821216116

Kulik LV, Dzuba SA, Grigoryev IA, Tsvetkov YD (2001) Electron dipoledipole interaction in ESEEM of nitroxide biradicals. Chem Phys Lett 343 (3-4):315-324. doi:Doi 10.1016/S0009-2614(01)00721-7

McCracken J (2011) Electron Spin Echo Envelope Modulation (ESEEM) Spectroscopy. In: Encyclopedia of Inorganic and Bioinorganic Chemistry. John Wiley \& Sons, Ltd. doi:10.1002/9781119951438.eibc0311

Meyer A, Abdullin D, Schnakenburg G, Schiemann O (2016) Single and double nitroxide labeled bis(terpyridine)-copper(II): influence of orientation selectivity and multispin effects on PELDOR and RIDME. Phys Chem Chem Phys 18 (13):9262-9271. doi:10.1039/c5cp07621h

Meyer A, Jassoy JJ, Spicher S, Berndhauser A, Schiemann O (2018) Performance of PELDOR, RIDME, SIFTER, and DQC in measuring distances in trityl based bi- and triradicals: exchange coupling, pseudosecular coupling and multi-spin effects. Phys Chem Chem Phys 20 (20):13858-13869. doi:10.1039/c8cp01276h

Meyer A, Schiemann O (2016) PELDOR and RIDME Measurements on a High-Spin Manganese(II) Bisnitroxide Model Complex. J Phys Chem A 120 (20):3463-3472. doi:10.1021/acs.jpca.6b00716

Milikisyants S, Scarpelli F, Finiguerra MG, Ubbink M, Huber M (2009) A pulsed EPR method to determine distances between paramagnetic centers with strong spectral anisotropy and radicals: The dead-time free RIDME sequence. J Magn Reson 201 (1):48-56. doi:10.1016/j.jmr.2009.08.008

Pliotas C, Dahl ACE, Rasmussen T, Mahendran KR, Smith TK, Marius P, Gault J, Banda T, Rasmussen A, Miller S, Robinson CV, Bayley H, Sansom MSP, Booth IR, Naismith JH (2015) The role of lipids in mechanosensation. Nat Struct Mol Biol 22 (12):991-998. doi: $10.1038 / \mathrm{nsmb} .3120$

Pliotas C, Ward R, Branigan E, Rasmussen A, Hagelueken G, Huang HX, Black SS, Booth IR, Schiemann O, Naismith JH (2012)

Conformational state of the MscS mechanosensitive channel in solution revealed by pulsed electron-electron double resonance (PELDOR) spectroscopy. P Natl Acad Sci USA 109 (40):E2675E2682. doi:10.1073/pnas.1202286109

Polyhach Y, Bordignon E, Tschaggelar R, Gandra S, Godt A, Jeschke G (2012) High sensitivity and versatility of the DEER experiment on nitroxide radical pairs at Q-band frequencies. Phys Chem Chem Phys 14 (30):10762-10773. doi:10.1039/c2cp41520h 
Schmidt T, Ghirlando R, Baber J, Clore GM (2016a) Quantitative Resolution of Monomer-Dimer Populations by Inversion Modulated DEER EPR Spectroscopy. Chemphyschem 17 (19):2987-2991. doi:10.1002/cphc.201600726

Schmidt T, Walti MA, Baber JL, Hustedt EJ, Clore GM (2016b) Long Distance Measurements up to 160 angstrom in the GroEL Tetradecamer Using Q-Band DEER EPR Spectroscopy. Angew Chem Int Edit 55 (51):15905-15909. doi:10.1002/anie.201609617

Spindler PE, Glaser SJ, Skinner TE, Prisner TF (2013) Broadband Inversion PELDOR Spectroscopy with Partially Adiabatic Shaped Pulses. Angew Chem Int Edit 52 (12):3425-3429. doi:10.1002/anie.201207777

Prisner T, Rohrer M, MacMillan F (2001) Pulsed EPR Spectroscopy: Biological Applications. Annual Review of Physical Chemistry 52:279-313. doi:10.1146/annurev.physchem.52.1.279

Valera S, Ackermann K, Pliotas C, Huang HX, Naismith JH, Bode BE (2016) Accurate Extraction of Nanometer Distances in Multimers by Pulse EPR. Chem-Eur J 22 (14):4700-4703. doi:10.1002/chem.201505143

Van Doorslaer S (2017) Hyperfine Spectroscopy: ESEEM. Emagres 6 (1):51-69. doi:10.1002/9780470034590.emrstm1517

Volkov A, Dockter C, Bund T, Paulsen H, Jeschke G (2009) Pulsed EPR Determination of Water Accessibility to Spin-Labeled Amino Acid Residues in LHCIIb. Biophys J 96 (3):1124-1141. doi:10.1016/j.bpj.2008.09.047

von Hagens T, Polyhach Y, Sajid M, Godt A, Jeschke G (2013) Suppression of ghost distances in multiple-spin double electronelectron resonance. Phys Chem Chem Phys 15 (16):5854-5866. doi: $10.1039 / \mathrm{c} 3 \mathrm{cp} 44462 \mathrm{~g}$

Ward R, Bowman A, Sozudogru E, El-Mkami H, Owen-Hughes T, Norman DG (2010) EPR distance measurements in deuterated proteins. J Magn Reson 207 (1):164-167. doi:10.1016/j.jmr.2010.08.002

Ward R, Pliotas C, Branigan E, Hacker C, Rasmussen A, Hagelueken G, Booth IR, Miller S, Lucocq J, Naismith JH, Schiemann O (2014) Probing the Structure of the Mechanosensitive Channel of Small Conductance in Lipid Bilayers with Pulsed Electron-Electron Double Resonance. Biophys J 106 (4):834-842. doi:10.1016/j.bpj.2014.01.008

Yang ZY, Liu YP, Borbat P, Zweier JL, Freed JH, Hubbell WL (2012) Pulsed ESR Dipolar Spectroscopy for Distance Measurements in 
Immobilized Spin Labeled Proteins in Liquid Solution. J Am

Chem Soc 134 (24):9950-9952. doi:10.1021/ja303791p

Zavoisky E (1945) Spin-magnetic resonance in paramagnetics. Journal of

Physics USSR 9:211-245 